\title{
Decrease in the frequency of treatment for patent ductus arteriosus after implementation of consensus guidelines: a 15-year experience
}

\author{
Lara Pavageau ${ }^{1}$ Luc P. Brion ${ }^{1}$ - Charles R. Rosenfeld $\mathbb{C D}^{1}$ - L. Steven Brown ${ }^{2}$. Claudio Ramaciotti ${ }^{3}$. \\ P. Jeannette Burchfield ${ }^{1} \cdot$ Mambarambath A. Jaleel $^{1}$
}

Received: 7 February 2019 / Revised: 22 May 2019 / Accepted: 3 June 2019 / Published online: 23 July 2019

(c) Springer Nature America, Inc. 2019

\begin{abstract}
Background Patent ductus arteriosus (PDA) management varies widely among neonatologists. Local problem Lack of institution-specific evidence-based guidelines for therapeutic closure of PDA.

Methods Quality improvement project among infants $<30$ weeks gestational age (GA) designed to determine whether the odds of therapy for closing the PDA, adjusted for GA, decreased after implementing evidence-based guidelines.

Intervention Implementation of guidelines with conservative approach to PDA management.

Results The frequency of PDA treatment decreased from 446/1125 (40\%) in Epoch 1 to 96/482 (20\%) in Epoch 2. PDA treatment was more frequent in neonates 23-26 weeks GA than those of 27-29 weeks GA (43\% vs. 28\%, respectively). Among 542 infants receiving indomethacin for PDA, 25\% had subsequent ligation; the odds of ligation after indomethacin were lower in neonates 27-29 weeks GA and decreased during Epoch 2.

Conclusions The frequency of medical and surgical treatment for therapeutic closure of PDA decreased after implementing evidence-based treatment guidelines.
\end{abstract}

\section{Introduction}

Despite the availability of an effective therapy, neonatologists continue to struggle with the optimal management of a patent ductus arteriosus (PDA) in the neonatal period. Although several investigators have examined the association of PDA with various neonatal morbidities, including chronic lung disease (CLD) [1], necrotizing enterocolitis (NEC) [2], and severe intraventricular hemorrhage (IVH) [3], the contribution of a PDA to these morbidities remains unclear. Moreover, no randomized controlled trial (RCT)

Supplementary information The online version of this article (https:// doi.org/10.1038/s41372-019-0432-8) contains supplementary material, which is available to authorized users.

Mambarambath A. Jaleel

mambarambath.jaleel@utsouthwestern.edu

1 Division of Neonatal-Perinatal Medicine, UT Southwestern Medical Center, Dallas, TX, USA

2 Parkland Health and Hospital System, Dallas, TX, USA

3 Division of Pediatric Cardiology, UT Southwestern Medical Center, Dallas, TX, USA has included a control group with watchful waiting for spontaneous closure with supportive care. Most RCTs have focused on the timing of early treatment rather than the risks of PDA closure versus complications following prolonged PDA patency [4, 5]. Furthermore, medical and surgical therapies for closing a PDA may have untoward side effects, such as renal, intestinal, and cardiovascular dysfunction, as well as neurodevelopmental impairment [6-9].

The associated morbidities and uncertainty of potential benefits of medical therapies for PDA closure reflect a wide practice variation. In the last 10 years, numerous investigators have reported a decrease in both medical and surgical intervention for PDA worldwide [10, 11]. More interestingly, although there was an overall decline in the percentage of neonates with a PDA who were treated with a COX inhibitor, this varied from 19 to $100 \%$ among centers. In addition, the percentage of infants undergoing surgical ligation was quite variable, ranging from 5 to $35 \%$ [10, 11].

Since nearly $40 \%$ of extremely low birth weight (ELBW; $<1000 \mathrm{~g}$ birth weight (BW)) and a larger proportion of very low birth weight neonates (VLBW; $<1500 \mathrm{~g} \mathrm{BW}$ ) experience spontaneous closure of the PDA by 1 week postnatal age, a more conservative approach to PDA management is warranted [12]. This is supported by recent studies in which 
Fig. 1 Flow diagram of consensus guidelines for PDA management that was implemented in 2010 at Parkland Hospital NICU. wks weeks, GA gestational age, BW birth weight, PDA patent ductus arteriosus, bpm beats per minute, vent ventilatory support, CXR chest radiograph, CT cardiothoracic, ECHO echocardiogram

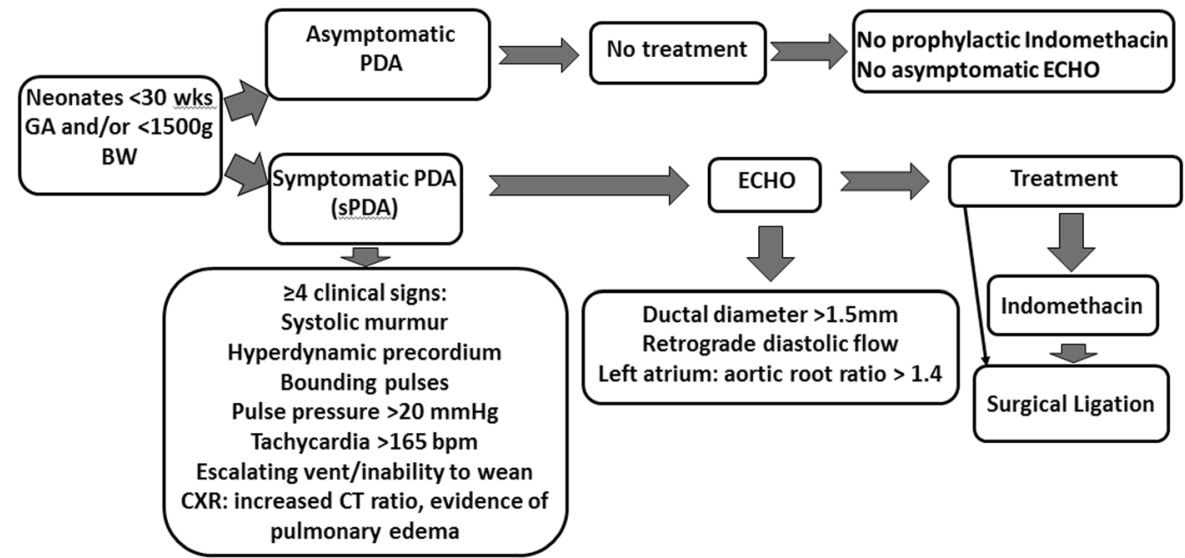

the likelihood of spontaneous closure was even higher, i.e., $60-97 \%$ in infants $<26$ weeks gestational age (GA) and/or VLBW at 44 days to 49 weeks postmenstrual age [13-15]. Importantly, no study to date has clearly observed an improvement in mortality, long-term respiratory, or neurodevelopmental outcomes following pharmacological treatment of a PDA.

In an effort to decrease variation in practice at Parkland Hospital, currently a 96-bed Level-III Neonatal Intensive Care Unit (NICU) with $>12,000$ deliveries/year and more than 35 faculty and 12 fellows, we implemented evidencebased consensus guidelines with a conservative approach to PDA management since 2010.

\section{Specific aims}

The objectives of this project were to examine the trend in PDA management in our NICU following implementation of consensus guidelines in 2010 and to determine if we were under-treating PDA and/or waiting too long before recommending surgical ligation following a more conservative approach to managing PDA.

\section{Methods}

\section{Study design}

This quality improvement (QI) project included a retrospective baseline period from January 01, 2001 through December 31, 2010 (Epoch 1) and a prospective period from January 01, 2011 through November 30, 2015 (Epoch 2), which followed implementation of consensus guidelines (Fig. 1) [16]. QI teams at Parkland Hospital included attending physicians, fellows, a database nurse and statistician. Cause-and-effect analysis disclosed several factors contributing to variation in PDA management in the NICU (Fig. 2). The plan-do-study-act cycle was started in
January 2011 and included education and encouragement to follow the consensus guidelines (Fig. 3). Though treatment teams in the NICU were expected to follow the guidelines, there were no processes to track ongoing protocol adherence and physician compliance was assessed retrospectively.

\section{Inclusion and exclusion criteria}

This QI project included all neonates born at $23+1 / 7$ and $29+6 / 7$ weeks GA, admitted to the NICU between 2001 and 2015 , and identified in a long standing validated prospective NICU database [17]. Neonates were grouped into two epochs, before (2001-2010, $n=1125)$ and after (2011-2015, $n=482$ ) implementation of consensus guidelines, and into two GA groups, 23-26 weeks and 27-29 weeks. One patient received indomethacin prophylaxis and was excluded from the study.

\section{Intervention}

The literature review and Parkland Hospital NICU data for PDA management of neonates $<30$ weeks GA and $<1500 \mathrm{~g}$ BW were discussed and reviewed every 5 years by all members of the Division of Neonatal-Perinatal Medicine at University of Texas Southwestern Medical Center (UTSW) and presented in the Division's Neonatal Critically Appraised Topic Conference. Evidence-based consensus guidelines [9, 16, 18, 19] were created (Fig. 1) and made readily available to all staff in the NICU within a web-based clinical decision support tool (NeoSource), with an icon link embedded within the electronic health record (EHR) platform. The first part of the guideline was to define a symptomatic PDA (SPDA) since there is a wide variation in the definition among institutions and clinicians. The definition of sPDA with cardiopulmonary compromise included clinical, radiographic, and echocardiographic evidence. The diagnosis was based on the criteria established by Cotton 
Fig. 2 Cause-and-effect diagram contribute to the variability in practice for PDA management in the NICU. NEC necrotizing enterocolitis, ARF acute renal failure, Echo echocardiogram, PDA patent ductus arteriosus demonstrating the factors that

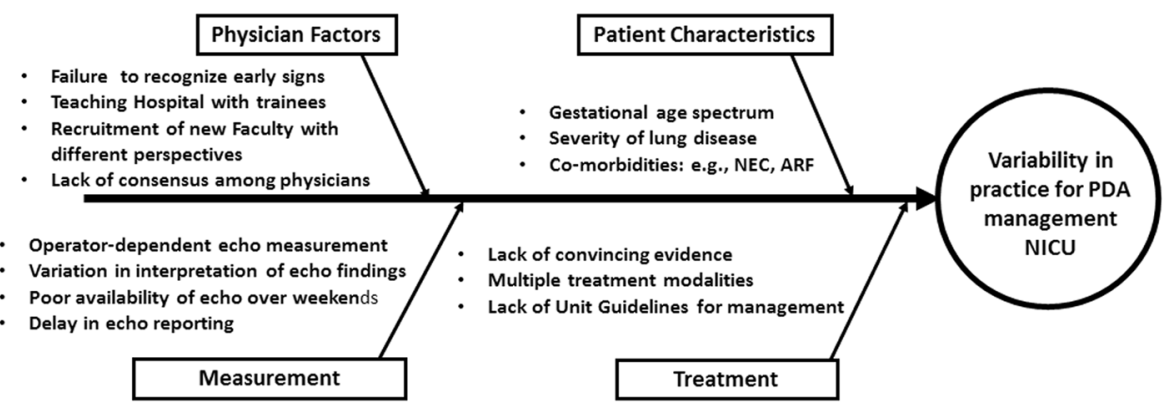

Interventions

Fig. 3 Key driver diagram for plan-do-study-act. PDA patent ductus arteriosus, GA gestational age, wks weeks, PHHS Parkland Health \& Hospital System, yrs years, NeoCAT Neonatal Critically Appraised Topic, EHR electronic health record

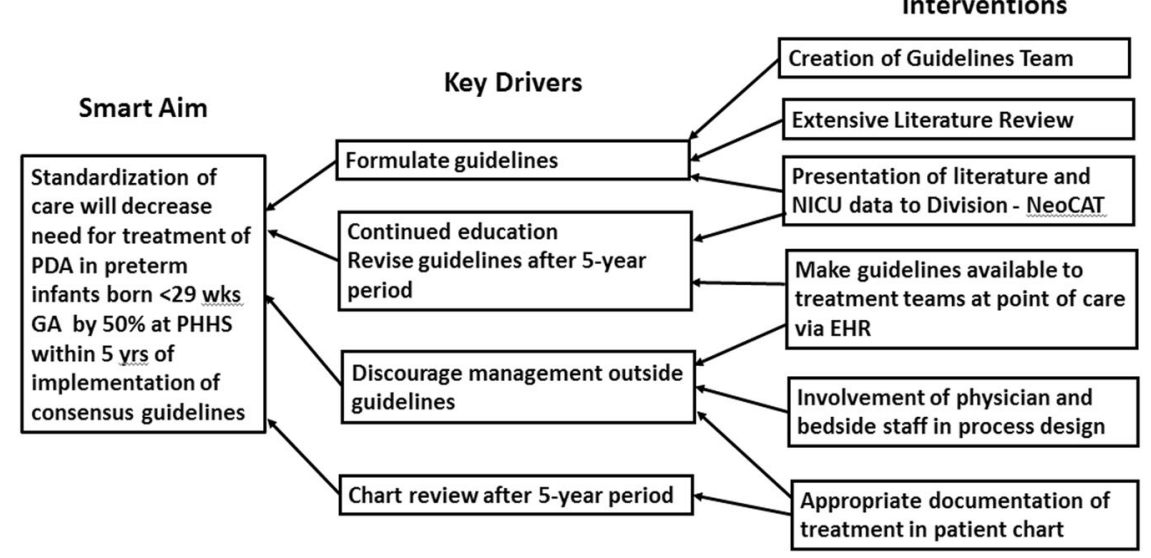

et al. [20] and required a large left-to-right shunt (i.e., at least four of the clinical signs not explained by another comorbid condition: tachycardia $>165$ beats/min, systolic murmur, hyperdynamic precordium, bounding peripheral pulses, widened pulse pressure $>20 \mathrm{mmHg}$, and escalating ventilator requirements or inability to wean ventilator support; chest X-ray showing increased cardiothoracic ratio and evidence of pulmonary edema and/or increased vascular markings) [21]. If the neonate was considered symptomatic, an echocardiogram was obtained and those neonates with ultrasonographic findings of a large PDA (ductal diameter $>1.5 \mathrm{~mm}$, retrograde diastolic flow in the descending aorta, and left atrium-to-aortic root ratio $>1.4$ ) were treated with intravenous indomethacin in the first 14 days of life. If the infant was $>14$ days old and/or had previously received indomethacin for an SPDA and remained symptomatic after 14 days of life, the infant was considered a candidate for surgical ligation if a repeat echocardiogram showed persistence of an SPDA. Conservative management to minimize left-to-right shunt often included supportive care at the discretion of the clinical team with fluid restriction, short course of diuretics, and/or distending airway pressure.

\section{Study of the intervention/measures/analysis}

Outcome variables included the percent of infants treated for a PDA with either indomethacin, surgical ligation, or both. The impact of our intervention was assessed by determining if the odds of therapy for PDA changed after implementation of the consensus guidelines and if the change was different in the two GA subgroups. Other variables, including GA, BW, indomethacin administration, ligation, age at ligation, and the diagnosis of a PDA were collected from the prospective NICU database [17]. Balancing measures included data on the duration of invasive and noninvasive ventilation, length of hospital stay, mortality, and the presence of any other comorbidity such as NEC stage II or more, severe IVH (Grades 3 and 4) and bronchopulmonary dysplasia (BPD). BPD was defined as an oxygen requirement after 36 weeks postmenstrual age (calculated from GA and date off supplementary oxygen without relapse for more than 1 day) if $<32$ weeks GA or 28 days if GA > 32 weeks [22]. Medical records of infants in Epoch 2 were reviewed for symptoms of an sPDA and compliance with consensus guidelines.

Another balancing measure was to determine whether surgical ligation had been inappropriately delayed after implementation of the guidelines. In order to accomplish this, a designated cardiologist (CR), who was blinded to clinical outcomes, retrospectively reviewed the presurgical echocardiograph of infants in Epoch 2 who underwent surgical ligation after indomethacin treatment. The CR assessed signs of pulmonary hypertension, such as the presence of at least one of the following: right ventricular 
hypertrophy, flattening of interventricular septum, presence of tricuspid regurgitation, and elevated right ventricular pressures [23], and/or presence of cardiovascular decompensation, such as abnormal ventricular function based on the left ventricular shortening fraction, low PDA gradient, and/or bidirectional or right-to-left shunt.

\section{Statistical analysis}

The changes in annual percentage of treatment for PDA closure over time were displayed using statistical process control charts (p-chart) using QI Macros ${ }^{\oplus}$ for Excel with mean and control limits calculated for each epoch. Continuous variables are presented as means $\pm \mathrm{SD}$ or median and 25th, 75th interquartile ranges. Demographic variables were compared between epochs using $\chi^{2}$ analysis, Student's $t$-test, and Mann-Whitney $U$ test, dependent on the variable type, using SPSS version 19 (IBM, Inc.). The binary multivariable logistic regression analysis determined whether the odds of therapy for closing the PDA, adjusted for GA group (23-26 weeks and 27-29 weeks, respectively), changed after implementing the guidelines. Statistical significance was asserted using two-tailed tests if $P<0.05$.

\section{Results}

During the study period, 2001-2015, there were 1607 deliveries of live neonates born at 23-29 weeks GA. Of these, $542(34 \%)$ received some form of therapy for a PDA and were included in the study, 446 in Epoch 1 and 96 in Epoch 2 (Table 1). After retrospective chart review, we found that on six occasions, infants received PDA treatment outside of the proposed guidelines in Epoch 2. This included infants on minimal respiratory support and/or insufficient documentation to conclude if they had sPDA.

There were no significant differences between the study groups for GA, BW, gender, race/ethnicity, or the complications associated with preterm birth except for a greater occurrence of RDS and use of surfactant in Epoch 2 (Table 1). The average annual percent of neonates with an sPDA receiving treatment decreased by $50 \%$ after implementing the new clinical guidelines (Fig. 4); the percent fell from 446/1125 (40\%) in Epoch 1 to 96/482 (20\%) in Epoch $2, P<0.001$. Over the 15 years, PDA treatment was less frequent in neonates 27-29 weeks GA than those of 23-26 weeks GA, 270/978 (28\%) versus 272/629 (43\%), respectively; $P<0.001$ (Supplementary Fig. 1). Treatment within each GA group decreased significantly after implementing the new clinical guidelines, 229/677 (34\%) to 41/ $301(14 \%)$ in neonates $27-29$ weeks GA and $217 / 448$ (48\%) to 55/181 (30\%) in neonates $23-26$ weeks GA, $P<$ 0.001 . The adjusted odds for PDA therapy were
Table 1 Characteristics of neonates who received PDA treatment during Epochs 1 and 2

\begin{tabular}{|c|c|c|c|}
\hline Characteristic & $\begin{array}{l}\text { Epoch } 1 \\
2001-2010 \\
(n=446)\end{array}$ & $\begin{array}{l}\text { Epoch } 2 \\
2011-2015 \\
(n=96)\end{array}$ & $P$-value \\
\hline Overall GA (weeks) & $27 \pm 2^{\mathrm{a}}$ & $26 \pm 1$ & NS \\
\hline Birth weight (grams) & $952 \pm 241^{\mathrm{a}}$ & $916 \pm 253$ & NS \\
\hline Female & $201(45)^{\mathrm{c}}$ & $50(52)$ & NS \\
\hline \multicolumn{4}{|l|}{ Race/ethnicity } \\
\hline White not Hispanic & $28(6)$ & $4(4)$ & \\
\hline Hispanic & 309 (69) & $63(66)$ & \\
\hline Black not Hispanic & $105(24)$ & $28(29)$ & \\
\hline Asian & $4(1)$ & $1(1)$ & \\
\hline Indomethacin only & $275(62)$ & $71(74)$ & 0.02 \\
\hline 23-26 weeks & $117(54)$ & $37(67)$ & NS \\
\hline 27-29 weeks & $158(69)$ & $34(83)$ & NS \\
\hline $\begin{array}{l}\text { Ligation after } \\
\text { Indomethacin failure }\end{array}$ & $120(27)$ & 16 (17) & 0.04 \\
\hline 23-26 weeks & $71(33)$ & $12(22)$ & NS \\
\hline 27-29 weeks & $49(21)$ & $4(10)$ & NS \\
\hline Ligation only & $51(11)$ & $9(9)$ & NS \\
\hline 23-26 weeks & $29(13)$ & $6(11)$ & NS \\
\hline 27-29 weeks & $22(10)$ & $3(7)$ & NS \\
\hline Age at ligation (days) & $16(9,24)^{\mathrm{b}}$ & $28(24,38)$ & $<0.01$ \\
\hline $\begin{array}{l}\text { Received antenatal } \\
\text { steroids }\end{array}$ & $207(46)$ & $36(38)$ & NS \\
\hline Received surfactant & $349(78)$ & $86(90)$ & $<0.01$ \\
\hline RDS & $390(87)$ & $94(98)$ & $<0.01$ \\
\hline Ventilation days & $11(3,28)$ & $11(2,28)$ & NS \\
\hline CPAP days & $22(8,35)$ & $32(19,44)$ & $<0.01$ \\
\hline $\begin{array}{l}\text { Days of positive airway } \\
\text { pressure (ventilation } \\
\text { and CPAP) }\end{array}$ & $38(20,62)$ & $49(29,67)$ & 0.02 \\
\hline Days on oxygen & $55(29,85)$ & $58(27,96)$ & NS \\
\hline Length of hospital stay & $94(70,120)$ & $83(61,111)$ & NS \\
\hline BPD & $263(59)$ & $64(67)$ & NS \\
\hline NEC (Stage II or more) & $45(10)$ & $8(8)$ & NS \\
\hline IVH (Grades 3 and 4) & $59(13)$ & $12(13)$ & NS \\
\hline Death & 45 (10) & $16(17)$ & NS \\
\hline
\end{tabular}

Data were analyzed by $t$-test or $\chi^{2}$

$G A$ gestational age, $R D S$ respiratory distress of newborn, $C P A P$ continuous positive airway pressure, $B P D$ bronchopulmonary dysplasia, $N E C$ necrotizing enterocolitis, IVH intraventricular hemorrhage

${ }^{\mathrm{a}}$ Means $\pm \mathrm{SD}$

${ }^{\mathrm{b}}$ Median (25th, 75th centiles) with Mann-Whitney test

${ }^{\mathrm{c}}$ Values in parenthesis are percent of values in the column

significantly lower in neonates $27-29$ weeks GA relative to those of 23-26 weeks GA (adjusted odds ratio [aOR] 0.50, 95\% confidence interval $[\mathrm{CI}]: 0.40,0.62 ; P<0.01)$ and decreased significantly during Epoch 2 relative to Epoch 1 (aOR 0.38, CI: $0.29,0.49 ; P<0.01$ ). Notably, the change in 
Fig. 4 Control chart (P-chart) showing serial annual percentages of infants treated for an SPDA. The frequency of PDA treatment decreased from $40 \%$ in Epoch 1 to 20\% in Epoch 2. PDA patent ductus arteriosus, UCL upper confidence limit, LCL lower confidence limit

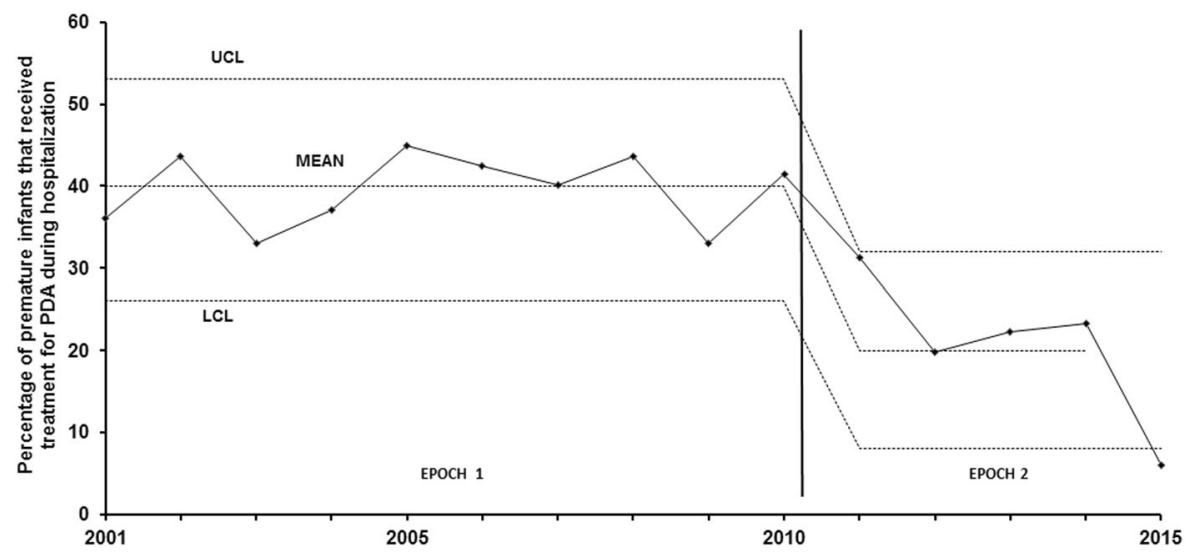

treatment across epochs did not differ between the two GA groups $(P=0.4)$.

The percentage of neonates who were treated with surgical ligation without prior exposure to indomethacin was similar in both epochs, 51/446 (11\%) and 9/96 (9\%). Among infants receiving indomethacin for PDA in both epochs, 136/542 (25\%) subsequently had surgical ligation; however infants in Epoch 2 had lower medication failure rate $(17 \%$ vs. $27 \%)$. The adjusted odds of ligation after medical failure were significantly lower in neonates 27-29 weeks GA relative to those of 23-26 weeks GA (aOR 0.54, CI: 0.36, 0.80; $P<0.01$ ) and decreased significantly in Epoch 2 (aOR 0.51, CI: 0.29, 0.91; $P=0.02$ ). Notably, the change in treatment from Epoch 1 to Epoch 2 did not differ between the two GA groups within those undergoing surgical ligation after failure $(P=0.3)$. Median age of ligation was $16(9,24)$ days in Epoch 1 versus 28 $(24,38)$ days in Epoch $2(P \leq 0.01)$.

Importantly, in the infants with an sPDA requiring treatment, the percent with BPD, NEC stage II or higher, severe IVH, and mortality, as well as the number of ventilated days, number of days on oxygen, and the length of hospital stay did not differ between epochs. There was an increase in the number of days on continuous positive airway pressure (CPAP) and the total number of ventilated plus positive airway pressure days during the hospitalization in Epoch 2 (Table 1). When a similar comparison was analyzed for the entire population of infants born between 23-29 weeks GA, the rates of BPD, NEC, IVH, ventilation, oxygenation, and hospitalization days, as well as, mortality were not significantly different in both epochs. The overall incidence of RDS, surfactant administration, and number of days on CPAP was noted to be higher in the second epoch (Supplementary Table 1).

All preoperative echocardiograms of the 27 neonates who underwent ligation in Epoch 2 had evidence of either a moderate or a large PDA; none had an abnormal shortening fraction; four of nine had a high diastolic flow in the left pulmonary artery; 6/23 had a low PDA gradient; and none had a bidirectional or right-to-left shunt. None had evidence of Eisenmeger syndrome or signs of pulmonary hypertension prior to surgical intervention.

\section{Discussion}

Clinicians have struggled with the PDA conundrum for many decades. The absence of a global consensus on optimal management, the reported morbidities associated with treatment, and the overwhelming evidence of spontaneous PDA closure have contributed to this dilemma. In an effort to standardize our care, we initiated a QI project in the NICU at Parkland Hospital designed to guide our staff in adopting a conservative evidence-based approach to managing a PDA. After implementing the guidelines, we observed a $50 \%$ decrease in therapeutic intervention in neonates $\leq 29$ weeks GA with either indomethacin and/or surgical ligation, regardless of GA stratum. Moreover, we neither observed evidence of clinical deterioration nor an increased incidence of the various morbidities and mortality after the consensus guidelines was implemented.

The wide practice variation emphasized the need for standardization of PDA treatment at our institution. The treatment teams in the NICU at Parkland Hospital rely heavily on the establishment of consensus protocols and guidelines to provide standardization of care due to the large number of trainees and medical providers. This is especially important when controversies and naturally occurring practice variations among clinicians are observed. Hence, we implemented detailed consensus guidelines using evidence-based approach to PDA management. Due to a lack of compelling neurodevelopmental follow-up data with ibuprofen use for PDA management, our guidelines standardized the use of indomethacin as drug of choice. Since existing evidence suggests that ductal ligation is associated with CLD, retinopathy of prematurity and poor neurodevelopmental outcomes [7-9], our guidelines reserved surgical intervention only for the pharmacotherapy 
refractory cases or if a neonate became symptomatic after 14 days of life. Therefore, the implemented guidelines were based on studies supporting feasible and safe conservative management of sPDA plus convincing data regarding the likelihood of spontaneous closure, the inefficacy in reducing mortality or comorbidities, the inadvertent side effects associated with treatment options, and the high incidence of pharmacological treatment failure [12-15, 18, 19, 24].

Once the new clinical guidelines were in place, the annual percentage of neonates treated for sPDA treatment decreased 50\% between 2001-2010 and 2011-2015. As expected, this decrease was greater in neonates 27-29 weeks GA than 23-26 weeks GA. Although neonates 27-29 weeks GA were less likely to receive therapy for a SPDA, the observed relative decrease in treatment in Epoch 2 occurred equally in both GA strata. Published data on spontaneous closure being more frequent in VLBW than in ELBW supports our finding and is a possible contributing factor [12-14]. Interestingly, the percentage of infants treated with surgical ligation without prior exposure to indomethacin did not differ between epochs. In contrast, the percentage of infants undergoing surgical ligation after failure of indomethacin therapy decreased $\sim 37 \%$ following introduction of the consensus guidelines. In earlier studies, up to $67 \%$ of neonates receiving indomethacin therapy were at risk of therapeutic failure [25]. In the present study, only $25 \%$ of neonates receiving indomethacin subsequently had surgical ligation of the PDA and thus might be classified as a treatment failure. Notably, the odds of ligation after medical failure were less in the older GA cohort. The odds of ligation after pharmacological treatment failure were also significantly lower in Epoch 2. More recently, multiple studies from the United States and Canada have observed similar decrease in the number of VLBW infants who received pharmacological and surgical intervention to close the PDA [26-28], but unlike our study, these did not establish a cause-effect relationship.

A major concern about the persistence of a PDA has been its contribution to neonatal morbidity, and/or the presence of respiratory or cardiovascular decompensation from an untreated sPDA [29, 30]. Our rates of BPD, IVH, NEC, and mortality were similar in the two epochs. This was true not only for those infants who needed medical and/ or surgical treatment for sPDA (Table 1) but also for the entire population of infants born at 23-29 weeks GA (Supplementary Table 1). This is consistent with the report given by Semberova et al. [15] showing unchanged rates of IVH, periventricular leukomalacia, BPD, and NEC in VLBW, and supports the argument that nonintervention management is relatively safe. They reported that only $6 \%$ of 297 eligible infants were treated to close the PDA, however, their criteria for treatment were not stated. Thus, unlike our study, they did not provide a guide for determining which neonates might benefit from therapeutic intervention. Others have also observed no increases in mortality or morbidity, i.e., BPD, periventricular leukomalacia, and NEC, in association with a decrease in the rate of ductus ligation following a conservative approach that tolerates the presence of a PDA as long as signs of cardiopulmonary compromise do not develop [31]. The increased occurrence of RDS in the second epoch for the infants with sPDA (78\% vs. $90 \%)$ and the entire population (71\% vs. $82 \%$ ) is noteworthy. In the infants with SPDA, this could be due to the selection of sicker patients for treatment as per the restrictive consensus guidelines. This could also be due to better documentation of RDS as a diagnosis after implementation of EHR in the second epoch.

Clinical decompensation causing cardiopulmonary compromise due to a delay in surgical ligation was a major concern and used as a balancing measure in our study. Notably, none of the neonates requiring surgical ligation in Epoch 2 had evidence of pulmonary hypertension or Eisenmeger syndrome on their echocardiogram, suggesting surgical intervention had not been inappropriately delayed. Other balancing measures showed a significant increase in the number of days on CPAP and in the total number of days on positive airway pressure days (the sum of days on mechanical ventilation and CPAP) during hospitalization, observed in Epoch 2. This could be attributed to the emphasis on noninvasive respiratory support as a primary mode of ventilation for the most immature infants.

\section{Strengths}

The change in percentage of PDA treatment establishes a functional relationship with implementation of consensus guidelines in 2010. The intervention was targeted at the system or organizational level and not at an individual level. The guidelines were available to the entire team within the EHR; this most likely influenced clinical practice without having to monitor individual physician compliance. Another strength of the study is the availability of a prospectively collected NICU database for $>15$ years by a single coordinator. This QI project is generalizable to other institutions and could be implemented if consensus is obtained with physicians in a group practice steering to improve neonatal care.

\section{Limitations}

The single center study spans over 15 years and may be influenced by overall improvement in NICU management over the last decades, i.e., our participation in the surfactant, positive pressure and oxygen RCT (SUPPORT) [32] and by changes in our clinical practice following the trial [33]. The introduction of noninvasive respiratory support as a primary 
mode of ventilation for the most immature infants, adoption of gentler forms of ventilation strategies, changes in initial fluid management, and optimization of nutrition also could influence PDA management. Another limitation might be the absence of a mechanism to enforce or track adherence to the guidelines. However, as mentioned in the strengths of the study, this process was intentionally designed to influence the practice at a system level and not scrutinize individual physician preference or compliance. After chart review, we concluded that on six occasions infants received PDA treatment outside the guidelines, though this was done retrospectively and hence relying on proper documentation of EHR. A third limitation is that our NICU database does not provide information on whether comorbidities like IVH or NEC occurred before or after PDA treatment. Also, the retrospective echocardiogram review for cardiopulmonary compromise was only done for those infants who underwent surgical ligation and the review occurred only in Epoch 2. Finally, an attempt to retrieve follow-up data on our patients was made; however, the complete dataset was not available due to the 15-year span of the study.

In summary, the implementation of evidence-based consensus guidelines was followed by a significant decrease in the frequency of therapeutic PDA closure among premature infants born at our institution. A protocolbased approach standardizes care when wide practice variation is prevalent for a common condition and provides clinicians with a structured framework for treatment. This is helpful until confirmation of the benefits of the conservative PDA management over early closure awaits a RCT.

Author contributions LP: participated in conceptualization and study design, data collection and interpretation, writing the first draft of the manuscript, and approving the final manuscript submitted. LPB: participated in conceptualization and study design, data interpretation, writing the first draft of the manuscript, and approving the final manuscript submitted. CRR: participated in conceptualization and study design, data interpretation, writing the first draft of the manuscript, and approving the final manuscript submitted. LSB: participated in conceptualization and study design, statistical analyses, data interpretation, writing the first draft of the manuscript, and approving the final manuscript submitted. CR: participated in interpretation of the echocardiogram findings, writing the first draft of the manuscript, and approving the final manuscript submitted. PJB: participated in data collection from the validated NICU database, writing the first draft of the manuscript, and approving the final manuscript submitted. MJ: participated in conceptualization and study design, data interpretation, writing the first draft of the manuscript, and approving the final manuscript submitted. Preliminary results were presented by LP at the annual meetings of Pediatric Academic
Societies May 2017, Hot Topics in Neonatology December 2017, and the Southern Society for Pediatric Research February 2018.

\section{Compliance with ethical standards}

Conflict of interest The authors declare that they have no conflict of interest.

Ethical approval This study was approved by the institutional review board of UTSW and by Parkland Hospital as a QI project and exempt from the need for informed consent.

Publisher's note: Springer Nature remains neutral with regard to jurisdictional claims in published maps and institutional affiliations.

\section{References}

1. Marshall DD, Kotelchuck M, Young TE, Bose CL, Kruyer L, O'Shea TM. Risk factors for chronic lung disease in the surfactant era: a North Carolina population-based study of very low birth weight infants. Pediatrics. 1999;104:1345-50.

2. Ryder RW, Shelton JD, Guinan ME. Necrotizing enterocolitis: a prospective multicenter investigation. Am J Epidemiol. 1980;112:113-23.

3. Dykes FD, Lazzara A, Ahmann P, Blumenstein B, Schwartz J, Brann AW. Intraventricular hemorrhage: a prospective evaluation of etiopathogenesis. Pediatrics. 1980;66:42-49.

4. Van Overmeire B, Van de Broek H, Van Laer P, Weyler J, Vanhaesebrouch P. Early versus late indomethacin treatment for patent ductus arteriosus in premature infants with respiratory distress syndrome. J Pedia. 2001;138:205-11.

5. Cotton RB, Stahlman MT, Berder HW, Graham TP, Catterton WZ, Kover I. Randomized trial of early closure of symptomatic patent ductus arteriosus in small preterm infants. J Pedia. 1978;93:647-51.

6. Jones LJ, Craven PD, Attia J, Thakkinstian A, Wright I. Network meta-analysis of indomethacin versus ibuprofen versus placebo for PDA in preterm infants. Arch Dis Child Fetal Neonatal Ed. 2011;96:F45-F52.

7. Weisz DE, McNamara PJ. Patent ductus arteriosus ligation and adverse outcomes: causality or bias? J Clin Neonatol. 2014;3:67-75.

8. Kabra NS, Schmidt B, Roberts RS, Doyle LW, Papile L, Fanroff A. Neurosensory impairment after surgical closure of patent ductus arteriosus in ELBW infants: results from the TIPP. Pediatrics. 2007;150:229-34.

9. Madan JC, Kendrick D, Hagadorn JI, Frantz IV. Patent ductus arteriosus therapy: impact on neonatal and 18-month outcome. Pediatrics. 2009;123:674-81.

10. Eunice Kennedy Shriver National Institute of Child Health and Human Development Neonatal Research Network. Generic database of very low birth weight infants (GDB). NCT00063063. http://www.clinicaltrials.gov/ct2/show/NCT00063063.

11. Laughon M, Bose C, Clark R. Treatment strategies to prevent or close a patent ductus arteriosus in preterm infants and outcomes. $\mathrm{J}$ Perinatol. 2007;27:164-70.

12. Koch J, Hensley G, Roy L, Brown S, Ramaciotti C, Rosenfeld $\mathrm{CR}$. Prevalence of spontaneous closure of the ductus arteriosus in neonates at a birth weight of 1000 grams or Less. Pediatrics. 2006;117:1113-21.

13. Rolland A, Shankar-Aguilera S, Diomande D, Zupan-Simunek V, Boileau P. Natural Evolution of patent ductus arteriosus in the 
extremely preterm infant. Arch Dis Child Fetal Neonatal. 2015;100:F55-8.

14. Sung SI, Chang YS, Chun JY, Yoon SA, Yoo HS, Ahn SY, et al. Mandatory closure versus nonintervention for patent ductus arteriosus in very preterm infants. J Pedia. 2016;177:66-71.

15. Semberova J, Sirc J, Miletin J, Kucera J, Berka I, Sebkova S, et al. Spontaneous closure of patent ductus arteriosus in infants $\leq 1500 \mathrm{~g}$. Pediatrics. 2017;140:e20164258.

16. Chiruvolu A, Jaleel MA. Therapeutic management of patent ductus arteriosus. Early Hum Dev. 2009;85:151-5.

17. Sanchez AM, Mize SG, Jimenez JM, Manroe BL, Rosenfeld CR, Tyson TE. Systems approach to the evaluation of maternal and neonatal care. In: Proceedings of the 12th Hawaii international conference on system sciences, selected papers in medical information processing. 1979. p. 140-51.

18. Furzan JA, Reishe J, Tyson JE, Laird P, Rosenfeld CR. Incidence and risk factors for symptomatic patent ductus arteriosus among inborn very-low-birth-weight infants. Early Hum Dev. 1985;12:39-48.

19. Mouzinho AI, Rosenfeld CR, Risser R. Symptomatic patent ductus arteriosus in very-low-birth-weight infants: 1987-1989. Early Hum Dev. 1991;27:65-77.

20. Cotton RB, Lindstrom DP, Stahlman MT. Early prediction of symptomatic patent ductus arteriosus from perinatal risk factors: a discriminant analysis model. Acta Paediatr. 1981;70:723-127.

21. Zonnenberg I, de Waal K. The definition of a haemodynamic significant duct in randomized controlled trials: a systematic literature review. Acta Paediatr. 2012;101:247-51.

22. Ehrenkranz RA, Walsh MC, Vohr BR, Jobe AH, Wright LL, Fanaroff AA, et al. Validation of the National Institutes of Health consensus definition of bronchopulmonary dysplasia. Pediatrics. 2005; $116: 1353-60$

23. Bhat R, Salas AA, Foster C, Carlo WA, Ambalavanan N. Prospective analysis of pulmonary hypertension in extremely low birth weight infants. Pediatrics. 2012;129:e682-9.
24. Clyman RI, Couto J, Murphy GM. Patent ductus arteriosus: are current neonatal treatment options better or worse than no treatment at all? Semin Perinatol. 2012;36:123-9.

25. Van Overmeire B, Smets K, Lecoutere D, Van de Broek H, Weyler J, De Groote K, et al. A comparison of ibuprofen and indomethacin for closure of patent ductus arteriosus. N Engl J Med. 2000;343:674-81.

26. Ngo S, Profit J, Gould J, Lee HC. Trends in patent ductus arteriosus diagnosis and management for very low birth weight infants. Pediatrics. 2017;139:e20162390.

27. Hagadorn JI, Brownell EA, Trzaski JM, Johnson KR, Lainwala S, Campbell BT, et al. Trends and variation in management and outcomes of very low-birth-weight infants with patent ductus arteriosus. Pedia Res. 2016;80:785-92.

28. Lokku A, Mirea L, Lee SK, Shah PS. On behalf of the Canadian Neonatal Network. Trends and outcomes of patent ductus arteriosus treatment in very preterm infants in Canada. Am J Perinatol. 2017;34:441-50.

29. Benitz WE. Committee on fetus and newborn, American Academy of Pediatrics. Patent ductus arteriosus in preterm infants. Pediatrics. 2016;137:1-6.

30. Elhoff JJ, Ebeling M, Hulsey TC, Atz AM. Potential unintended consequences of a conservative management strategy for patent ductus arteriosus. Congenit Heart Dis 2016;11:52-7.

31. Jhaveri N, Moon-Grady A, Clyman RI. Early surgical ligation versus a conservative approach for management of PDA that fails to close after indomethacin treatment. J Pediatrics. 2010;157:381-7.

32. Carlo WA, Finer NN, Walsh MC, Rich W, Gantz MG, Laptook AR, et al. Target ranges of oxygen saturation in extremely preterm infants. N Engl J Med. 2010;362:1959-69.

33. LeVan JM, Brion LP, Wrage LA, Gantz MG, Wyckoff MH, Sanchez PJ, et al. Change in practice after the surfactant, positive pressure and oxygenation randomized trial. Arch Dis Child Fetal Neonatal Ed. 2014;99:F386-F39. 